\title{
Effect of the antitussive glaucine on bronchomotor tone in man
}

\author{
O CONSTANT, B SLAVIN, JR LEHANE, C JORDAN, JG JONES
}

From the Division of Respiratory Medicine, Northwick Park Hospital, and the Division of Anaesthesia, MRC Clinical Research Centre, Harrow; and the Royal Free Hospital School of Medicine, London

\begin{abstract}
In view of the observation that the antitussive agent glaucine prevents histamineinduced bronchoconstriction in guinea pigs we investigated this agent for a possible peripheral action in man, using a new method for measuring changes in bronchomotor tone. The forced airflow oscillation method was used to determine respiratory resistance $\left(R_{r s}\right)$ over a range of lung volumes $(\mathrm{VL})$ in seven healthy supine subjects. Computer analysis of the hyperbolic relationship between $R_{\mathrm{rs}}$ and $\mathrm{VL}$ was used to determine the asymptotic resistance and yield estimates of lower airways conductance (Glaw). Specific lower airways conductance (sGlaw) was expressed as the slope of the linear plot of Glaw against VL and is a sensitive index of bronchomotor tone. After baseline measurements of sGlaw subjects received placebo or $60 \mathrm{mg}$ glaucine orally acording to a double-blind crossover protocol. Histamine, $500 \mu \mathrm{g}$, was inhaled 45 minutes later. Measurements of sGlaw were repeated every 10 minutes for two hours. Although there was a trend towards bronchodilatation after glaucine administration (sGlaw $=130 \%$ of baseline) there was no significant difference from the effect of placebo (sGlaw $=89 \%$ of baseline). After inhalation of histamine sGlaw fell to $26 \%$ of baseline after both glaucine and placebo $(p<0.01)$. In a further study three subjects received glaucine and placebo according to an identical protocol except that the histamine was omitted. Again the increase in sGlaw failed to achieve significance. Glaucine does not affect the bronchoconstrictor response to histamine in man and there is no convincing evidence of an effect on resting bronchomotor tone.
\end{abstract}

Glaucine (Lepetit) is an alkaloid obtained from the plant Glaucium flavum Crantz (Papaveraceae) and has been used for many years as a cough suppressant in eastern Europe. Although it bears a chemical resemblance to the opiates its antitussive effect is not reversed by naloxone and it lacks other opiate characteristics. ${ }^{1}$

Drugs may suppress cough by different mechanisms. Cough is usually a vagally mediated reflex initiated by stimulation of irritant receptors in the respiratory tract. Some antitussive drugs may exert their effects by depressing the cough reflex at brainstem level. Other drugs, such as local anaesthetic agents, may act on the peripheral part of this reflex arc at vagal afferent nerve endings in the respiratory tract. Observations made by Corrao et $\mathrm{al}^{2}$ suggest an

Address for reprint requests: Dr JG Jones, Northwick Park Hospital, Harrow, Middx, HA1 3UJ.

Accepted 8 February 1983 alternative peripheral action. They reported some cases in which intractable cough was eliminated promptly by bronchodilator treatment, and when this was discontinued three to 12 months later the cough returned. This suggests that modification of the tone of airways smooth muscle may regulate the sensitivity of irritant receptors in the airways. Thus antitussive activity may be mediated in three ways: (a) a central effect; (b) through a peripheral action on bronchomotor tone, increasing the threshold for the firing of the irritant receptors; and (c) a direct effect on irritant receptors without any effect on resting bronchomotor tone.

Elucidation of mechanisms of action may permit the development of agents which are free of undesirable central effects. Furthermore, antitussive agents may on occasion be indicated in patients with airways obstruction or bronchial hyperreactivity and knowledge of the effects of these drugs on airway calibre may thus be of value to the clinician. Since glaucine has been shown to reduce the effects of 
histamine on the lung in guinea pigs, ${ }^{3}$ we investigated the possibility that this agent may have a peripheral action by comparing the effects of glaucine and placebo on resting bronchomotor tone before and after the administration of histamine. Bronchomotor tone was measured by a specially developed technique to obtain specific conductance of the lower airways (sGlaw) from measurements of respiratory resistance. ${ }^{4}$

\section{Methods}

\section{SUBJECTS}

Ten healthy male volunteers aged $21-44$ years were studied. Each subject was studied on two separate occasions, receiving either DL-glaucine 1,5-bisphosphate $60 \mathrm{mg}$ orally or a similarly flavoured placebo according to a double-blind randomised crossover protocol. The study was approved by the Northwick Park Hospital ethical committee, and informed consent was obtained from each subject.

\section{RESISTANCE-VOLUME PLOTS}

Plots of total respiratory resistance $\left(R_{r s}\right)$ against change in lung volume were obtained as the subjects breathed out slowly $\left(31 \mathrm{~min}^{-1}\right)$ from 1 litre above functional residual capacity (FRC) to residual volume with the use of the apparatus shown in figure 1. The forced airflow oscillation method ${ }^{56}$ was used to measure $R_{r s}$ during the expiratory manoeuvre. The oscillating airflow was produced by a purpose-built

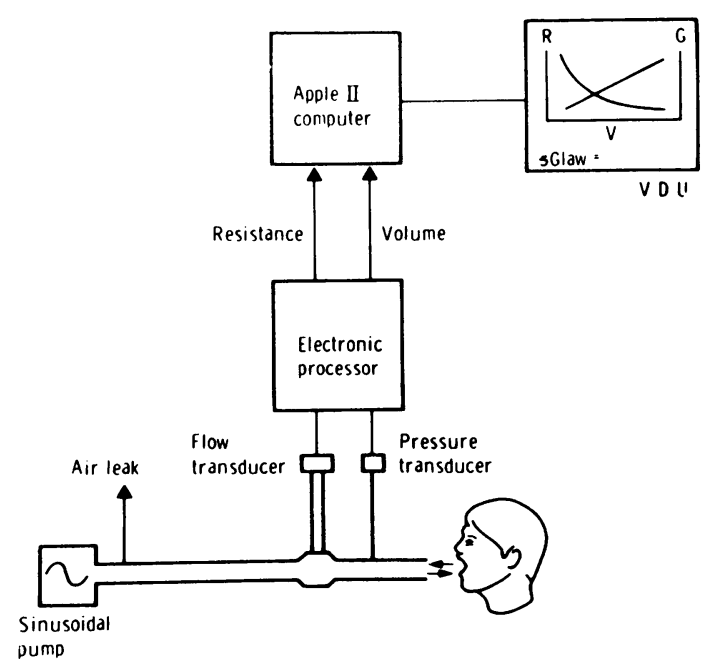

Fig 1 Apparatus for determining specific lower airways conductance from measurements of respiratory resistance over a range of lung volumes. Reproduced by courtesy of the British Journal of Anaesthesia. " sinusoidal pump (developed by the division of bioengineering of the MRC Clinical Research Centre at Northwick Park Hospital) delivering a stroke volume of $58 \mathrm{ml}$ at a frequency of $3 \mathrm{~Hz}$. The flow signal was obtained with a heated Fleisch No 1 pneumotachograph and differential pressure transducer (Validyne MP 45). Mouth pressure was obtained with a Pye Ether UP2 transducer. The pressure and flow signals were analysed electronically and $R_{r s}$ and change in lung volume from FRC (VL) were derived. About $50 R_{r s}-V_{L}$ co-ordinates were obtained during each expiratory manoeuvre.

\section{SPECIFIC LOWER AIRWAYS CONDUCTANCE}

It has been shown that the relationship between lower airways conductance and lung volume is linear, ${ }^{7}$ and that the slope of this relationship (sGlaw) can be used as a sensitive index of bronchomotor tone. ${ }^{4}$ To obtain lower airways resistance (and thus lower airways conductance) from $R_{r s}$ it is necessary to subtract the resistances of upper airway, lung tissue, and chest wall (that is, those components of $R_{r s}$ which do not tend towards zero as lung volume increases). A computer-aided curvefitting routine was therefore used to fit a hyperbola of the type: $1 /\left(R_{\mathrm{rs}}-R_{\mathrm{A}}\right)=$ sGlaw $\times\left(V_{L}+\right.$ ERV $)$. This equation has three parameters: asymptotic resistance $\left(R_{A}\right)$ asymptotic volume (ERV), and sGlaw, which are represented graphically in figure 2 .

$R_{A}$ represents that part of $R_{r s}$ which does not vary with lung volume (upper airway, lung tissue, and chest wall), so that $\left(R_{r s}-R_{A}\right)$ is the resistance of the distensible intrathoracic airways (the lower airways), and $1 /\left(R_{r s}-R_{A}\right)$ is the conductance of these airways. sGlaw is the slope of the plot of the conductance of the lower airways against $\mathrm{VL}$ and is in this method derived directly from the analysis of the hyperbolic plot of $R_{r s}$ and VL. ERV is here defined as the volume at which $R_{r s}$ would be infinite and may differ from the standard spirometric expiratory reserve volume.

\section{PROCEDURE}

The study was divided into two parts, $(a)$ and $(b)$.

(a) Seven subjects were studied in the supine position two hours after a light breakfast. Baseline measurements of sGlaw were obtained six times over a 30-minute period, after which each subject received either glaucine or placebo. Duplicate measurements of sGlaw were made at 10-minute intervals for $\mathbf{4 5}$ minutes. Then $500 \mu \mathrm{g}$ histamine was given by inhalation from a metered-dose aerosol and measurements were made for a further 75 minutes.

(b) To study possible effects of glaucine on resting bronchomotor tone occurring later than 45 


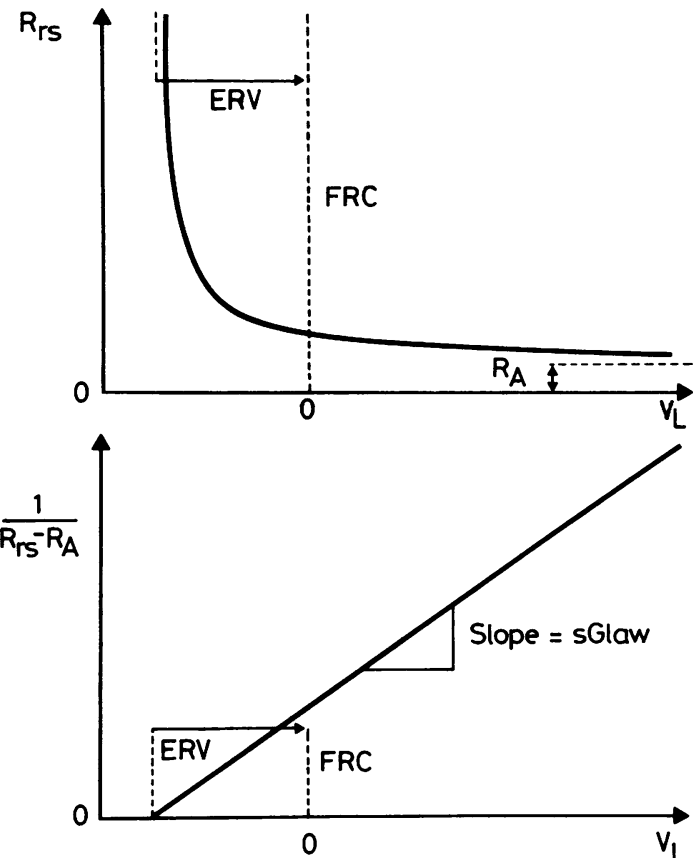

Fig 2 Top: General resistance $\left(R_{r s}\right)$-volume $(V L)$ hyperbola with asymptotes $R_{A}$ and $E R V$. Bottom: Linear plot of $1 /\left(R_{r s}-R_{A}\right)$ against volume with slope equal to specific lower airways conductance. (See text under "Methods.") (Reproduced by courtesy of the British Journal of Anaesthesia. " )

minutes, three other subjects were studied with glaucine or placebo by the same procedure described above but without the histamine challenge. sGlaw measurements were continued for 125 minutes after administration of glaucine or placebo.

\section{STATISTICAL ANALYSIS}

Logarithmic transformation was applied to the sGlaw data to correct for positive skew in distribution. This also corrected for the association between the level of sGlaw and degree of change observed. ${ }^{4}$ Analysis of variance allowing for repeated measures $^{8}$ was applied to the logarithmic data.

\section{Results}

(a) Representative curves showing $R_{\mathrm{rs}}$ plotted against $V_{L}$ and sGlaw in one subject are shown in figure 3. This shows the excellent fit of the curves to the data points and indicates the changes in shape of the $R_{r s}-V_{L}$ curves and the concomitant changes in slope of the conductance-volume lines (sGlaw) seen in this subject. For the group as a whole, baseline values for $R_{r s}$ and sGlaw were similar to previously reported normal values derived by this method ${ }^{4}$ with geometric mean sGlaw $=4.83 \mathrm{kPa}^{-1}$ $\mathrm{s}^{-1}$ (mean $\log _{\mathrm{e}} \mathrm{sGlaw}=-0 \cdot 73$, SD 0.38). After the administration of glaucine sGlaw rose to $130 \%$ of the control mean (fig 4), although analysis of variance showed that the results after glaucine administration were not significantly different from placebo values. After administration of histamine there was a significant reduction if sGlaw with both glaucine and placebo $(p<0.01)$, in both cases there was a significant reduction in sGlaw with both There was no significant difference in this response between glaucine and placebo and there was no difference between the recovery curves for placebo and glaucine (fig 4).

(b) In the second study, on the effects of glaucine and placebo alone, there was again a tendency for sGlaw to be higher, suggesting a reduction in resting bronchomotor tone after administration of glaucine, although this trend also failed to achieve significance (fig 5).

No untoward effects of glaucine were observed in the study.

\section{Discussion}

Glaucine, 30-60 mg given orally, has been shown to be an effective antitussive in man, ${ }^{9}$ producing peak plasma levels about 45 minutes after administration (JDF Lockhart, personal communication). It also reduces the effect of histamine on airway pressure in mechanically ventilated guinea pigs and this suggests a possible peripheral mechanism of action. Our study has produced no evidence for such a peripheral action in man.

The failure to detect a bronchodilator effect or any protective effect against histamine-induced bronchoconstriction in our subjects is unlikely to be due to insufficient sensitivity of the method used. The small degree of scatter in the placebo data shown in fig 4 in relation to the magnitude of the change produced by an aerosol of only $500 \mu \mathrm{g}$ histamine illustrates the sensitivity of this method. We have previously shown that it is at least as sensitive as the plethysmographic method for specific conductance. ${ }^{4}$ In healthy subjects only small decreases in resting bronchomotor tone are possible and we have shown that this method can detect these small changes when they are produced by rimiterol ${ }^{4}$ and salbutamol aerosols. ${ }^{10}$

The absence of any protective effect of glaucine against histamine-induced bronchoconstriction is unlikely to be related to the dose of histamine used. Although a weak bronchodilator effect may not be 


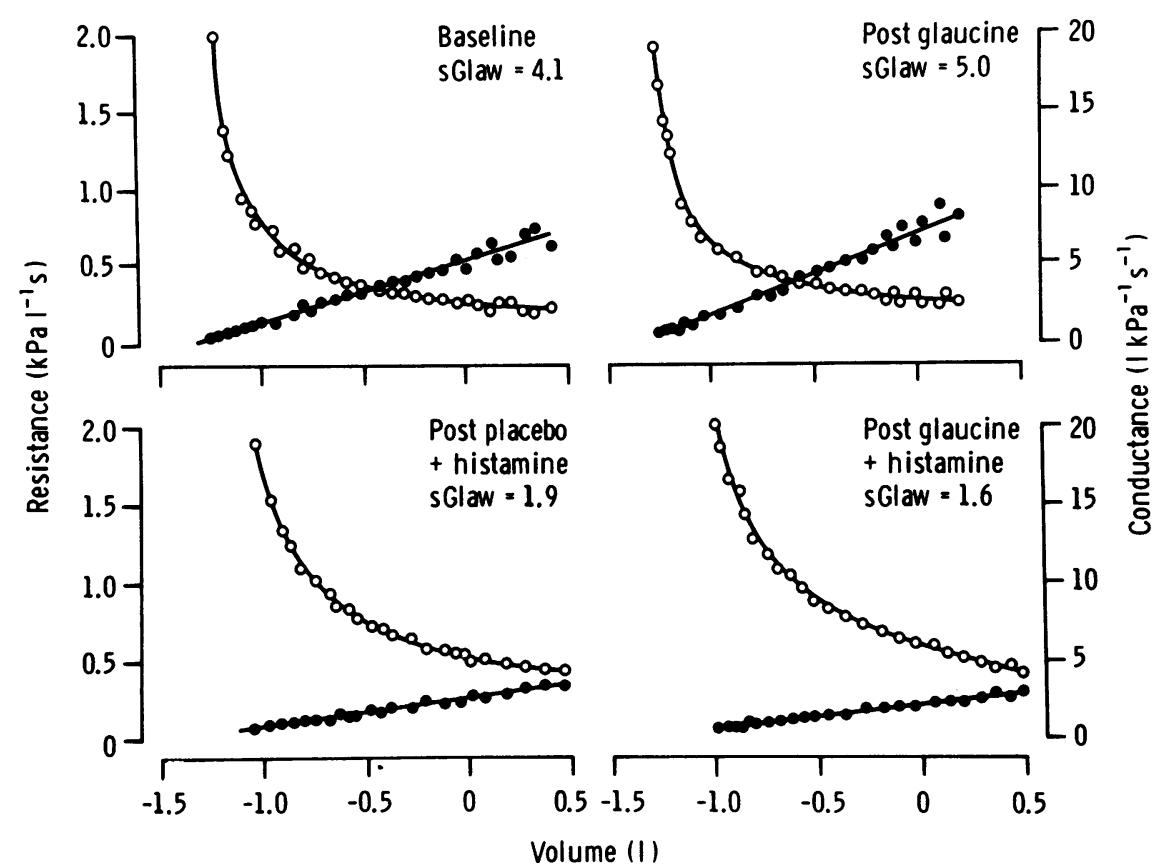

Fig 3 Computer plots showing resistance-volume data points $(O)$ with fitted curve and the corresponding lower airways conductance-volume data (๑), to illustrate the effects of glaucine and histamine in one of the subjects. These plots were drawn directly from the computer output.

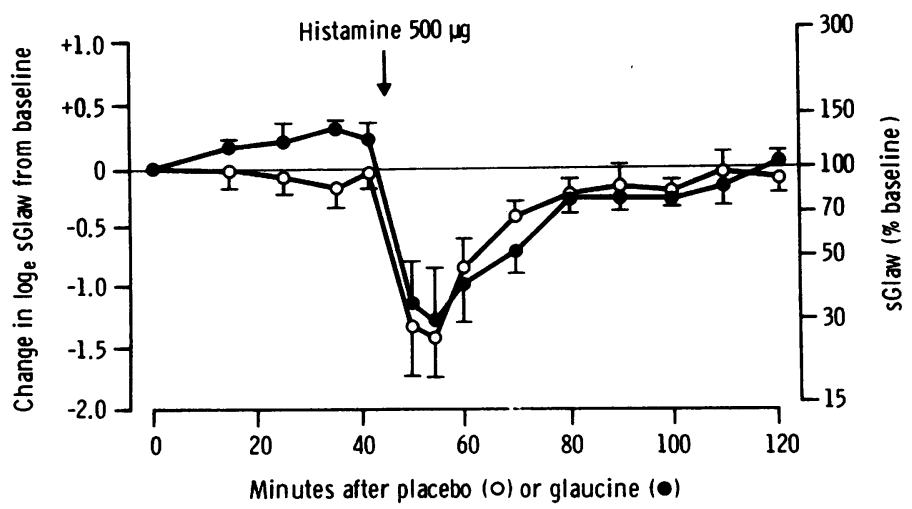

Fig 4 Mean changes ( \pm SEM) from baseline specific lower airways conductance (sGlaw) after administration of glaucine $(\Theta)$ and placebo $(O)$ with histamine challenge. The left $Y$ axis indicates difference in $\log _{e} s G l a w$ and the right $Y$ axis indicates percentage change in sGlaw. 


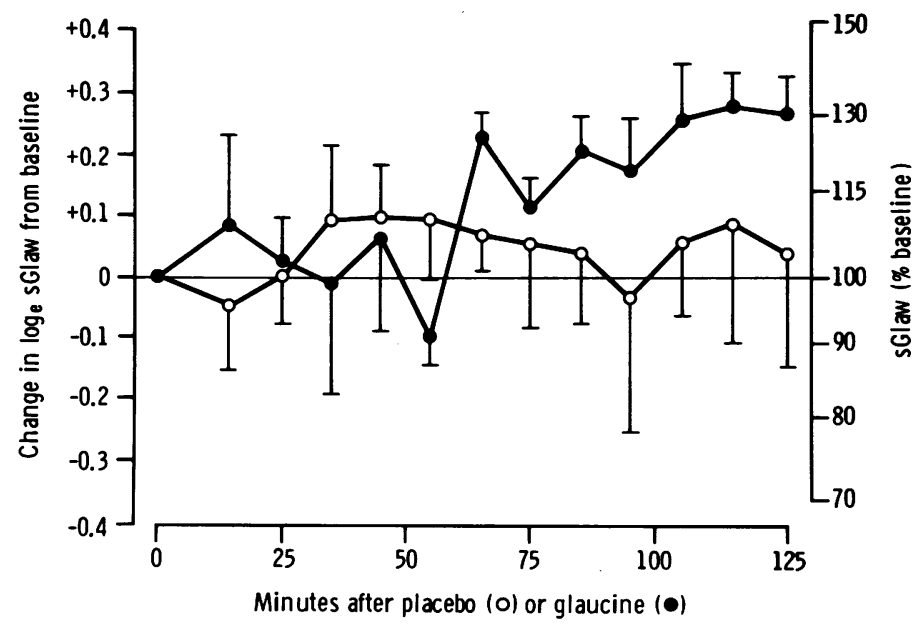

Fig 5 Mean changes ( \pm SEM) in sGlaw in three subjects after administration of glaucine and placebo without histamine challenge (symbols and scales as in fig 4).

sufficient to counteract a strong bronchoconstrictor effect, histamine $500 \mu \mathrm{g}$ by aerosol is a relatively small dose for healthy volunteers and produced only mild chest symptoms. The bronchoconstriction produced by this dose of histamine can be partly prevented by aminophylline ${ }^{10}$ and completely abolished by salbutamol ${ }^{10}$ and rimiterol. ${ }^{4}$

If a change in bronchomotor tone, which may indirectly affect the threshold of irritant receptors, is not the mechanism whereby glaucine inhibits cough it is still possible that glaucine has a peripheral action by directly depressing the sensitivity of the irritant receptors in the airways. If this were the case then it might be anticipated that it would reduce the bronchoconstrictor effect of histamine without affecting resting bronchomotor tone. No such effect was seen in this study, although a further study using other irritant substances, such as citric acid aerosol, may help to clarify this point, as the effects of histamine on bronchomotor tone which we observed may have been due to a direct effect on bronchial smooth muscle rather than a reflex effect.

This study illustrates the usefulness of this nonplethysmographic method for determining the reactivity of airway smooth muscle in pharmacological studies. The method was originally devised to enable such measurements to be made in passive subjects (that is, anaesthetised or mechanically ventilated patients); thus it is also applicable to animal studies on airway pharmacology. One of the main advantages of measuring specific lower airways conductance by this method is that in assessments of change in bronchomotor tone in intact animals and man it eliminates the effect of changes in airway calibre which occur as a result of changes in lung volume. ${ }^{11}$

In conclusion, glaucine did not reduce the bronchoconstrictor response to histamine and although there was a trend towards bronchodilatation before administration of histamine this failed to achieve significance. No evidence was found for a peripheral action of this antitussive agent. No deleterious effects of bronchomotor tone were observed in this study and there were no untoward side effects.

We would like to thank Lepetit Pharmaceuticals Ltd for supplies of glaucine and Riker Laboratories Ltd for the metered-dose histamine aerosols.

\section{References}

${ }^{1}$ Schuster CR, Aigner T, Johanson CE, Gieske TH. Experimental studies of the abuse potential of $\mathrm{d}, 1-$ glaucine 1.5 phosphate in rhesus monkeys. Pharmac Biochem Behav 1982;16:851-4.

${ }^{2}$ Corrao WM, Braman SS, Irwin RS. Chronic cough as the sole presenting manifestation of bronchial asthma. $N$ Engl J Med 1979;300:633-7.

${ }^{3}$ Kase Y, Kawaguchi M. Pharmacological studies on D-L glaucine phosphate as an antitussive. Arzneim Forsch (in press).

4 Jordan C, Lehane JR, Jones JG, Altman DG, Royston JP. Specific conductance using forced airflow oscillation in mechanically ventilated human subjects. J Appl Physiol 1981;51:715-24.

s Goldman M, Knudson RJ, Mead J, Peterson N, Schwaber JR, Wohl ME. A simplified measurement of respiratory resistance by forced oscillation. J Appl Physiol 1970;28:113-6. 
${ }^{6}$ Hyatt RE, Zimmerman IR, Peters GM, Sullivan WJ. Direct writeout of total respiratory resistance. J Appl Physiol 1970;28:675-8.

${ }^{7}$ Blide RW, Kerr DH, Spicer WS. Measurement of upper and lower airway resistance and conductance in man. $J$ Appl Physiol 1964;19:1059-69.

${ }^{8}$ Winer BJ. Statistical principles in experimental design. 2nd ed. Tokyo: McGraw-Hill, Kogakusha Ltd., 1971:514-603.

9 Dierckx P, Leblanc G, Decoster A, Criscuolo D. Double blind study of glaucine in chronic cough. Int J Clin Pharmacol 1981;19:396-9.

${ }^{10}$ Lehane JR, Slavin B, Jordan C, Jones JG. Aminophylline increases resting bronchomotor tone but reduces the bronchoconstriction response to histamine in healthy subjects. Am Rev Respir Dis 1982;125:225.

" Lehane JR, Jordan C, Jones JG. Influence of halothane and enflurane on respiratory airflow resistance and specific conductance in anaesthetised man. $B r J$ Anaesth 1980;52:773-80. 